\title{
6 ESTIMACIÓN DE LA COMPRENSIBILIDAD EN PANELES DE MUSEOS
}

\section{Measuring the readability of exhibit panels in museums}

\section{Jorge Morato, Sonia Sánchez-Cuadrado y Paolo Gimmelli}

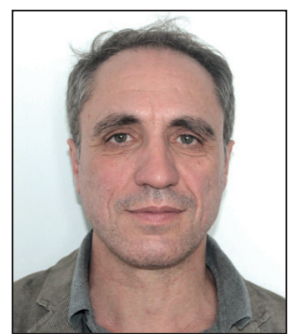

Jorge Morato inició los estudios de doctorado en 1993 y en 1999 defendió su tesis doctoral en Documentación en la Universidad Carlos III de Madrid. Desde el año 2000 es profesor de los estudios de Biblioteconomía y Documentación e Informática. Ha dirigido proyectos docentes y de investigación, tanto públicos como privados, sobre gestión de la información. Lidera el grupo de investigación GigaBD y dirige el Plan nacional de l+D+i para la mejora de métricas de comprensión de la información pública.

http://orcid.org/0000-0002-7530-9753

Universidad Carlos III de Madrid, Departamento de Informática Av. Universidad, 30. 28911 Leganés (Madrid), España jmorato@inf.uc3m.es

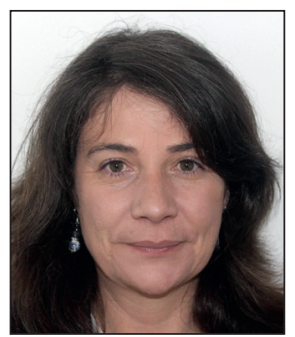

Sonia Sánchez-Cuadrado, doctora en Documentación, es profesora e investigadora en el Departamento de Biblioteconomía y Documentación de la Universidad Complutense de Madrid. Fue directora del Master Oficial Universitario en eLearning y Tecnología Educativa en el centro universitario Bureau Veritas Formación. Ha impartido clases en la Universidad Carlos III de Madrid en Documentación, Informática, Estadística y Administración y Dirección de Empresas. Su actividad investigadora está enfocada a los sistemas de organización del conocimiento, recuperación de información, extracción de información, reconocimiento de patrones y procesamiento del lenguaje natural actualmente aplicado a las métricas de comprensión de la información.

http://orcid.org/0000-0002-7722-1982

Universidad Complutense de Madrid, Departamento de Biblioteconomía y Documentación Santisima Trinidad, 37. 28010 Madrid, España sscuadrado@ucm.es

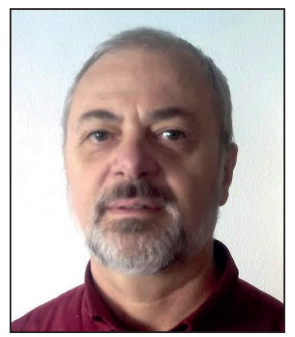

Paolo Gimmelli estudió Lenguas Modernas en el Istituto Universitario Orientale de Nápoles y es doctor en Lingüística Aplicada por la Universidad de Castilla-La Mancha (UCLM). Desde 1998 es profesor de italiano en la Escuela Oficial de Idiomas de Ciudad Real, y desde 2008 lo compagina con la docencia universitaria en la UCLM como profesor asociado. Su principal área de investigación es la enseñanza del italiano como lengua extranjera y la comprensión de la información. http://orcid.org/0000-0002-9485-7368

Universidad de Castilla-La Mancha, Departamento de Filología Moderna, Centro de Lenguas Ronda de Toledo, s/n, 13071 Ciudad Real, España paologimmelli@gmail.com

\section{Resumen}

Se evalúa la comprensibilidad de textos en español en paneles de museos, considerando que están dirigidos a todos los públicos y escritos en lenguaje estándar. Se recopilan los indicadores propuestos en la bibliografía científica sobre la comprensibilidad y se analiza su aplicación a los paneles de museos. Se ha construido un corpus con los textos de paneles de seis museos y se evalúa la dificultad de los paneles mediante las métricas clásicas y tests sobre la percepción del usuario. Con métodos de aprendizaje automático se analiza la capacidad de estas métricas clásicas para pronosticar la dificultad para el usuario. Se han añadido indicadores lingüísticos y de familiaridad del término para mejorar la precisión del pronóstico. Lo más eficaz para predecir el grado de comprensibilidad es un modelo híbrido de indicadores clásicos, lingüísticos y de familiaridad con los términos.

\section{Palabras clave}

Comprensibilidad; Lecturabilidad, Métricas; Museos; Paneles informativos; Procesamiento del lenguaje; Lista frecuencia terminológica. 


\begin{abstract}
The objective of this paper is the assessment of the comprehensibility in museum panels, considering that they are addressed to all audiences and written in standard language. The indicators proposed in the scientific bibliography on comprehensibility are compiled. Subsequently, their application to the museum panels is analyzed. A corpus is built with the texts of panels from six museums and the difficulty of the panels is evaluated by means of classical metrics and tests on users' perception. The ability of these classic metrics to predict the difficulty for the user is computed with automatic learning methods. Linguistic and term familiarity indicators have been added to improve the accuracy of the assessment. The most effective way to predict the degree of comprehensibility is a hybrid model of classical, linguistic and term familiarity indicators.
\end{abstract}

\title{
Keywords
}

Readability; Legibility; Metrics; Museum; Informative panels; NLP; Word frequency lists.

Morato, Jorge; Sánchez-Cuadrado, Sonia; Gimmelli, Paolo (2018). “Estimación de la comprensibilidad en paneles de museos". El profesional de la información, v. 27, n. 3, pp. 570-581.

https://doi.org/10.3145/epi.2018.may.10

\section{Introducción}

La lectura fácil, la accesibilidad cognitiva o el diseño universal son algunos conceptos asociados a la comprensión lectora. Estas iniciativas se están implantando incluso de modo normativo. En el caso particular de España existen dos leyes a favor de la comprensión de la información:

- la Ley 34/2002 sobre servicios de la sociedad de la información y comercio electrónico (España, 2002) establece que la documentación administrativa debe contener "información clara, comprensible e inequívoca";

- la Ley 19/2013 (España, 2013) en sus principios generales establece que toda la información debe ser comprensible conforme al principio de acceso universal y diseño para todos.

Estos requisitos ya fueron destacados por la IFLA en 1997 (Nomura; Nielsen; Tronbacke, 2010). Desde la organización propusieron unas directrices internacionales para materiales de lectura fácil dirigidas a la integración de personas con discapacidad y lectores con limitada competencia lingüística o lectora. Desde entonces se están recopilando pautas para los redactores y gestores de contenido, con el fin de facilitar la lectura y hacerla comprensible.

En lectura web, los usuarios apenas conceden unos segundos para valorar si determinado contenido resuelve su necesidad de información; si no lo ven claro buscan otro recurso. Por ello, en diseño web se proponen recomendaciones que faciliten la comprensión de los textos. Trabajar en contenidos de calidad expresados de forma clara compensa en cuanto en cuanto mejora el posicionamiento web (Morato et al., 2013).

Algo similar ocurre en los museos. Según el Laboratorio Permanente de Público de Museos del Ministerio de Educación se estima que los visitantes raramente permanecen más de 70 minutos en un gran museo, y que el tiempo dedicado a las obras que se observan, no suele sobrepasar los 30 segundos (Carbon, 2017).

La comprensión lectora es un proceso complejo, condicionado por múltiples factores como la capacidad de procesar la lectura, el bagaje educativo-cultural, y el diseño de la in- formación que se lee. La población española entre 16 y 65 años obtiene unas tasas bajas de comprensión lectora con respecto al resto de la Unión Europea (OECD, 2016). Según el informe Programme for the International Assessment of Adult Competencies (Piaac) de la OECD, el lector español tiene dificultades para extraer conclusiones y se pierde en un texto de cierta profundidad.

http://www.oecd.org/skills/piaac

Esto repercute de forma directa y negativa en la forma de transmitir la información. Dada esta realidad social nos proponemos identificar los indicadores de comprensibilidad de la información que permitan definir la facilidad/dificultad de la lectura de un texto.

Las iniciativas sobre lectura fácil y diseño universal deben extenderse más allá de la integración social de los discapacitados o el fomento de la lectura

\subsection{Comprensibilidad lectora}

Los términos comprensibilidad y legibilidad (en inglés understandability y readibility/legibility) han originado cierta controversia en la comunidad científica. La comprensibilidad o lecturabilidad se corresponde con la capacidad de comprender un texto (Sigaud-Sellos, 2010). Para algunos autores, la legibilidad engloba a ambos conceptos (Ferrando-Belart, 2004), mientras que en otros dominios, como en diseño web, la legibilidad se reserva casi exclusivamente para el aspecto externo o formato de un texto (contraste entre fondo y la letra, tamaño de fuente, etc.).

\subsection{Indicadores clásicos para medir la comprensibili- dad de los textos}

Las métricas clásicas surgidas en torno a los años 50 para estimar la comprensibilidad de los textos en inglés se basan en indicadores sobre el número de caracteres, sílabas, palabras, oraciones y párrafos. Este es el caso de las métricas de Flesch-Kinkaid, Gunning-Fog, Coleman-Liau Index, SMOG 
Tabla 1. Indicadores y métricas clásicas de comprensibilidad

\begin{tabular}{|c|c|c|c|c|c|c|c|c|c|}
\hline \multirow[b]{2}{*}{ Indicador } & \multicolumn{5}{|c|}{ Inglés } & \multicolumn{4}{|c|}{ Español } \\
\hline & Flesch & Gunning & Coleman & SMOG & ARI & FH & SP & L $\mu$ & Inflesz \\
\hline Total de palabras & & & & & & & . & . & . \\
\hline -N. palabras por oración & • & - & & & - & . & & & \\
\hline -Palabras de 3 o más sílabas & & . & & . & & & & & \\
\hline Total oraciones & & & & & & & . & & . \\
\hline -Oraciones cada 100 palabras & & & . & & & & & & \\
\hline Total sílabas & & & & & & & - & & \\
\hline -Sílabas por palabra & • & & & & & • & & & \\
\hline Media caracteres por palabra & & & & & & & & . & \\
\hline -Caracteres por palabra & & & & & . & & & & \\
\hline -Media caracteres cada 100 palabras & & & . & & & & & & \\
\hline -Varianza caracteres palabra & & & & & & & & . & \\
\hline
\end{tabular}

Index o Automated readability index (ARI) (Van-Oosten; Tanghe; Hoste, 2010).

Existen hasta 78 versiones de estas métricas, incluidas algunas para lenguas romances como el español, francés o italiano (Social Science Consulting, 2013). Las versiones de estas medidas clásicas adecuadas para al español son: Inflesz, Legibilidad $\mu(\mathrm{L} \mu)$, cálculo de Gutiérrez Polini, Flesh-Fernández Huerta (FH) o Szigriszt-Pazos (SP).

La tabla 1 muestra los indicadores utilizados en cada una de las métricas tanto para el inglés como para el español. El resultado establece la comprensibilidad de los textos por niveles y los valores dependen de cada métrica. Por ejemplo, en Fernández-Huerta (FH), la escala de niveles es: 1) muy fácil, 2) fácil, 3) algo fácil, 4) normal, 5) algo difícil, 6) difícil, y 7) muy difícil. Otras métricas como Inflesz tienen cinco valores: 1) muy fácil, 2) fácil, 3) normal, 4) difícil y 5) muy difícil.

\section{Los indicadores clásicos no son suficien-} tes para estimar la complejidad del texto

Estas métricas han sido diseñadas a partir de textos para determinar los niveles de lectura en educación. Buena parte de ellas resultan de la aplicación directa de la Ley de Zipf, donde las palabras más frecuentes tienden a ser más breves en número de caracteres. El uso frecuente de una palabra evidencia que una comunidad mayor de usuarios comprende el significado de la misma.

Estos indicadores y métricas no están exentos de limitaciones y críticas, en especial cuando los umbrales no están adaptados a las lenguas y contextos. Ejemplos de términos como álveo = lecho del río (con 5 caracteres) frente a revolucionario (con 14 caracteres) sirven para rebatir la afirmación sobre que la brevedad de las palabras implique necesariamente una mayor facilidad de comprensión. En este sentido, los investigadores Dale y Chall (1948) presentaron una alternativa al número de caracteres/sílabas por palabra basada en un indicador que recoge la familiaridad del término. De este modo, propusieron un listado de palabras simples o unigramas con la que presumiblemente podían predecir el nivel educativo del alumno.

\subsection{Indicadores lingüísticos para medir la comprensi- bilidad de los textos}

Más recientemente los estudios han incluido indicadores focalizados en elementos lingüísticos. François y Miltsakaki (2012) afirmaron que existen evidencias que demuestran que incluir técnicas de procesamiento del lenguaje natural (PLN) mejora las estimaciones de la comprensibilidad. Los factores se distribuyen entre los principales niveles del lenguaje: morfológico, sintáctico, léxico-semántico y pragmático.

1) Nivel morfológico: se analiza la categoría gramatical de las palabras. Morato et al. (2003) midieron la legibilidad y etiquetas lingüísticas para la identificación de textos científicos. En el trabajo de Zeng-Treitler et al. (2007) se compara el número de palabras desambiguadas Part of Speech (POS) por oración de tres corpus:

- informes médicos de un hospital;

- textos fáciles obtenidos de sitios web de salud;

- textos difíciles extraídos de artículos científicos en Medline.

Se clasifican los documentos como fácil o difícil estableciendo umbrales por categorías:

- verbos: entre los valores 2,06 - 3,21 lo considera fácil y entre los valores 1,41-2,29 difícil (a más verbos, los textos son etiquetados como más fáciles);

- nombres: 4,75 fácil frente a 5,48 difícil;

- nombres propios: 1,27 fácil, frente a 3,01 difícil.

2) Nivel sintáctico: en el estudio de Feng et al. (2010) se estudiaron aspectos de PLN (sintaxis, POS, densidad de entidades, o marcadores del discurso) con una muestra de estudiantes de primer grado. Los estudios de Graesser, McNamara y Kulikowich (2011) indican que los textos con menos complementos, menos palabras, y menos términos antes del verbo principal resultan más sencillos. En general, la simplicidad sintáctica favorece la comprensibilidad y está condicionada por diversos indicadores como el número de complementos por oración, número de palabras por 
Tabla 2. Indicadores utilizados para evaluar la dificultad de la comprensibilidad (+ uso, y - uso)

\begin{tabular}{|c|c|c|}
\hline Nivel & Indicador & Recogido en la bibliografía por \\
\hline \multirow{3}{*}{$\begin{array}{l}\text { Indicadores } \\
\text { clásicos }\end{array}$} & + Caracteres por palabra & $\begin{array}{l}\text { Zeng-Treitler et al., } 2007 \\
\text { François; Fairon, } 2012 \text { (para francés) }\end{array}$ \\
\hline & + Palabras por frase/longitud de oración & $\begin{array}{l}\text { Stenner, 1996; Zeng-Treitler et al., 2007; Leroy; Endicott, 2011; Graess- } \\
\text { er; McNamara; Kulikowich, } 2011 \\
\text { François; Fairon, } 2012 \text { (para francés) } \\
\text { Aluisio et al., } 2010 \text { (para portugués) } \\
\text { Venturi et al., } 2015 \text { (para italiano) }\end{array}$ \\
\hline & + Frases por párrafo & $\begin{array}{l}\text { Graesser et al., 2004; Zeng-Treitler et al., } 2007 \\
\text { François; Fairon, } 2012 \text { (para francés) }\end{array}$ \\
\hline \multirow{6}{*}{ Morfológico } & - Conectores causales & Graesser et al., 2004; Graesser; McNamara; Kulikowich, 2011 \\
\hline & - Acrónimos y abreviaturas no estándar & Temnikova; Vieweg; Castillo, 2015 \\
\hline & + Adjetivos & Leroy; Endicott, 2011 \\
\hline & + Nombres propios & Zeng-Treitler et al., 2007 \\
\hline & + Presencia de símbolos o notaciones especializadas & Zeng-Treitler et al., 2007 \\
\hline & + Sustantivos & Zeng-Treitler et al., 2007; Leroy; Endicott, 2011; 2012 \\
\hline \multirow{2}{*}{ Sintáctico } & - Simplicidad sintáctica & $\begin{array}{l}\text { Graesser; McNamara; Kukikowich, 2011; Kauchak; Leroy; Hogue, } 2017 \\
\text { François; Fairon, } 2012 \text { (para francés) }\end{array}$ \\
\hline & $\begin{array}{l}\text { + Complejidad del sintagma nominal (número palabras } \\
\text { en el sintagma) }\end{array}$ & Leroy; Endicott, 2011; 2012 \\
\hline \multirow{5}{*}{$\begin{array}{l}\text { Léxico-se- } \\
\text { mántico }\end{array}$} & + Acepciones del término & Graesser et al., 2004 \\
\hline & + Dispersión uso palabras del dominio & Stenner; Smith; Burdick, 1983 \\
\hline & $\begin{array}{l}\text { + Palabras infrecuentes/familiaridad. } \\
\text { Familiaridad terminológica (Palabras de uso infrecuente) }\end{array}$ & $\begin{array}{l}\text { Stenner; Smith; Burdick, 1983; Stenner, 1996; Elhadad, 2006; Ze- } \\
\text { ng-Treitler et al., 2007; Keselman et al., 2007; Leroy; Endicott, 2011; } \\
\text { 2012; Leroy et al., } 2013 \\
\text { François; Fairon, } 2012 \text { (para francés) }\end{array}$ \\
\hline & + Uso de conceptos abstractos & Stenner; Smith; Burdick, 1983; Graesser; McNamara; Kulikowich, 2011 \\
\hline & + Terminología especializada & Elhadad, 2006 \\
\hline \multirow[b]{2}{*}{ Pragmático } & - Cohesión profunda. La acción no sigue un hilo conductor & Graesser et al., 2004; Graesser; McNamara; Kulikowich, 2011 \\
\hline & - Cohesión referencial & $\begin{array}{l}\text { Graesser et al., 2004; Graesser; McNamara; Kulikowich, } 2011 \\
\text { François; Fairon, } 2012 \text { (para francés) }\end{array}$ \\
\hline
\end{tabular}

oración, o número de palabras previas al verbo principal. Pitler y Nenkiova (2008) combinaron varias características sintácticas (cohesión y marcadores del discurso, sintaxis, o número de términos), apoyándose adicionalmente en las métricas clásicas de comprensibilidad.

3) Nivel léxico-semántico: la información léxico-semántica de las palabras también influye en la comprensibilidad lectora. Se mide mediante frecuencias que establecen la familiaridad de la palabra, o el uso de terminología especializada recogida en recursos como tesauros y taxonomías (Elhadad, 2006), o el número de acepciones de una palabra.

4) Nivel pragmático: es un factor complejo que valora la frecuencia de un término y el uso de sinónimos. Se ha demostrado que la coherencia entre frases facilita la lectura (Graesser et al., 2004; Newbold; McLaughlin; Gillam, 2010), así como los indicadores de cohesión como los pronombres y las anáforas (Pitler; Nenkova, 2008). Otro de los factores influyentes es la distancia entre términos, ya sean palabras sueltas o compuestas, que pueden reflejar digresiones hacia otras ideas (Blanco; Lioma, 2012).

En relación con las métricas para la comprensión lectora, una revisión de la bibliografía científica permite mostrar un listado de indicadores sobre distintos aspectos del lenguaje.
Las tablas 2 y 3 recogen los indicadores usados en estudios previos, y si su presencia (+) o no (-) ha proporcionado criterios para facilitar o dificultar la comprensión lectora.

\subsection{Técnicas y recursos de evaluación para la com- prensibilidad}

La evaluación de la comprensión lectora se caracteriza por una amplia variedad de técnicas e instrumentos, aunque no está carente de limitaciones. Se puede medir la comprensión local y referencial mediante pruebas objetivas o semiobjetivas que se inician con la conexión proposicional que genera la microestructura de la base del texto y así establecer el hilo conductor y la cohesión referencial.

Un segundo nivel de comprensión está relacionado con la capacidad de asignar significado coherente y global al texto leído construyendo la macroestructura del texto (Montanero-Fernández, 2004). La percepción de la dificultad de la comprensión se suele evaluar mediante tests a los usuarios para que indiquen el nivel de dificultad (Kauchak; Leroy; Hogue, 2017).

De forma automática, el programa Coh-metrix (Graesser; McNamara; Kukikowich, 2011) examina y mide la coherencia de un texto en inglés. Este software analiza la cohesión 
Tabla 3. Indicadores utilizados para evaluar la facilidad de la comprensibilidad (+ uso, y - uso)

\begin{tabular}{|c|c|c|}
\hline Plano & Indicador & Recogido en la bibliografía por \\
\hline \multirow{11}{*}{ Morfológico } & - Nombres propios & Zeng-Treitler et al., 2007 \\
\hline & - Signos de puntuación & Zeng-Treitler et al., 2007 \\
\hline & - Sustantivos & Zeng-Treitler et al., 2007; Leroy; Endicott, 2012 \\
\hline & - Verbos modales & Zeng-Treitler et al., 2007 \\
\hline & + Adjetivos & Zeng-Treitler et al., 2007 \\
\hline & + Adverbios & Zeng-Treitler et al., 2007 \\
\hline & + Determinantes & Zeng-Treitler et al., 2007 \\
\hline & + Frecuencia de verbos & Zeng-Treitler et al., 2007; Leroy; Endicott, 2011; 2012; Leroy et al., 2013 \\
\hline & + Palabras funcionales & Leroy; Endicott, 2011; 2012; Leroy et al., 2013 \\
\hline & + Posesivos & Zeng-Treitler et al., 2007 \\
\hline & + Pronombres & $\begin{array}{l}\text { Graesser et al., 2004; Zeng-Treitler et al., } 2007 \\
\text { François; Fairon, } 2012 \text { (para francés) }\end{array}$ \\
\hline Léxico-semántico & - Palabras infrecuentes/familiaridad & Leroy; Endicott, 2012 \\
\hline \multirow[t]{2}{*}{ Sintáctico } & + Simplicidad sintáctica & $\begin{array}{l}\text { Graesser; McNamara; Kulikowich, 2011; Leroy; Endicott, } 2012 \\
\text { François; Fairon, } 2012 \text { (para francés) } \\
\text { Tonelli; Tran-Manh; Pianta, } 2012 \text { (para italiano) }\end{array}$ \\
\hline & + Frases verbales & Leroy et al., 2013 \\
\hline \multirow{3}{*}{ Pragmático } & + Cohesión profunda & Graesser et al., 2004 \\
\hline & + Cohesión referencial & $\begin{array}{l}\text { Graesser et al., 2004; Graesser; McNamara; Kulikowich, } 2011 \\
\text { François; Fairon, } 2012 \text { (para francés) } \\
\text { Tonelli; Tran-Manh; Pianta, } 2012 \text { (para italiano) }\end{array}$ \\
\hline & + Texto narrativo & Graesser; McNamara; Kulikowich, 2011 \\
\hline
\end{tabular}

referencial, la variedad léxica, la especificidad de los términos, los conectores lingüísticos, la complejidad/simplicidad sintáctica y el número de patrones. Coh-metrix dispone de versiones para otros idiomas como el portugués (Aluisio; Gasperin, 2010) y el italiano (Tonelli; Tran-Manh; Pianta, 2012). La versión para el español está en desarrollo.

A parte de Coh-metrix, existen otros recursos online para calcular la comprensibilidad. Readable.io tiene en cuenta métricas clásicas y proporciona consejos para mejorar la legibilidad. La herramienta SAM mide y muestra la información gráfica, siendo la única que lo considera. Se trata de un aspecto interesante, aunque según los autores Badarudeen y Sabharwal (2010) presenta un alto grado de subjetividad. La evaluación sólo de las métricas clásicas también puede realizarse a través de los recursos web Online-utility.org (para el inglés) y Legible.es (para el español).

https://legible.es

\section{Metodología. Material y métodos}

En este trabajo nos proponemos medir el nivel de comprensibilidad de textos en español. Limitamos el estudio a un tipo de texto breve destinado a ser leído en poco tiempo, de ámbito general y dirigido a todos los públicos. Queda fuera de los límites de este estudio la simplificación del texto (Bott et al., 2012) y la evaluación de los indicadores de legibilidad, entendida como la capacidad de ser legible, en cuanto a formato o presentación; contraste y tamaño de fuente determinado; también queda al margen la información gráfica. Con estas condiciones, se ha elaborado un estudio de caso que permitiese examinar los indicadores para la compren- sibilidad de los textos y medir el nivel de dificultad para un público objetivo amplio.

\subsection{Muestra}

En el contexto de los museos, la información descriptiva de los paneles expuestos en las exposiciones posee algunas características singulares acordes con las premisas del estudio como:

- describir elementos muy específicos de dominios concretos;

- disponer de un espacio y tiempo reducido para transmitir la información;

- amplio número de elementos a mostrar en algunas vitrinas con un único cartel;

- visitantes con niveles diferentes de comprensibilidad, diferente capacidad lectora y bagaje cultural, etc.

El tiempo medio que dedican los visitantes a leer un panel se estima en 12 segundos, según una observación a 30 personas en los museos visitados para este trabajo y realizada por los autores.

Para la elaboración de la muestra se acudió a los museos y, con el consentimiento del personal de las exposiciones, se fotografiaron al azar paneles con información descriptiva:

- Museo Arqueológico Nacional, Madrid;

- Museo Nacional de Antropología, Madrid;

- Museo de la Biblioteca Nacional, Madrid;

- Museo de la Historia, Madrid;

- Museo Egipcio, Barcelona;

- Museo Modernista, Barcelona. 
En aquellos casos en los que no se permitía el uso de cámaras se nos remitió a la información publicada en la web del museo (p. e. Museo del Prado, Museo Romántico). Sin embargo, tras examinar la información de la exposición virtual, estos casos han sido desestimados para este estudio, porque eran significativamente diferentes a los de la exposición física y dejaban de cumplir alguna de las premisas.

De los carteles fotografiados se seleccionó una muestra de 50 paneles (entre 7 y 10 paneles por museo con el fin de balancear el conjunto). Se procesaron todas las imágenes y se extrajo el texto mediante reconocimiento automático de caracteres (OCR). Al finalizar este proceso se realizó una revisión manual para asegurar la corrección del resultado.

Como en posicionamiento web, los paneles de museos deben transmitir la información en unos pocos segundos

\subsection{Recogida de datos y medidas}

En este trabajo se recogieron tres conjuntos de datos para valorar la comprensibilidad de los textos:

- valoración de comprensión de los participantes;

- evaluación basada en métricas clásicas;

- valores para los indicadores lingüísticos.

En primer lugar se recogieron los datos relativos a las métricas de comprensibilidad clásicas (FH, SP y L $\mu$ ) para el español. Se procesaron los 50 textos de la muestra con Legible.es que considera los indicadores clásicos como el número de palabras, oraciones, sílabas y caracteres (tabla 1).

En segundo lugar se hizo un test de comprensibilidad a 7 participantes con el perfil típico del visitante (adultos de más de 35 años con formación académica universitaria) de acuerdo con los datos del Ministerio de Cultura. Según los resultados del estudio, el perfil medio del público de museos es de mayoría femenino y de aproximadamente unos 42 años, español y con estudios universitarios (Ministerio de Cultura, 2010).

Para esta evaluación manual se utilizaron las directrices indicadas por Montanero-Fernández (2004). Se les ofreció el texto sin formato para que lo evaluasen. Los participantes realizaron un resumen que definía la idea principal. Además respondieron a cuestiones sobre la percepción de si les había resultado fácil o difícil de entender, de acuerdo con esos dos valores:

- fácil si eran capaces de identificar la idea principal y de recordar algunos datos;

- difícil si se perdían en la coherencia del discurso, no siendo capaces de reconocer la idea principal ni los datos relevantes mencionados.

En tercer y último lugar se recopilaron los datos para los indicadores de carácter lingüístico. Con ayuda de la herramienta de PLN Freeling (Padró et al., 2010), se volvieron a procesar los 50 textos y se anotó la categoría morfológica de cada palabra.

http://nlp.lsi.upc.edu/freeling/node/1
Las variables analizadas fueron:

- número de oraciones;

- número de palabras;

- número de párrafos;

- número de palabras compuestas;

- longitud de la oración;

- adjetivos calificativos y ordinales;

- adverbios;

- verbos condicionales;

- subjuntivos;

- sustantivos;

- nombres propios;

- pronombres personales, indefinidos y de relativo.

Adicionalmente se extrajeron los datos para los indicadores mediante expresiones regulares, y se determinó la familiaridad del término con los valores de frecuencia de uso de Freeling, OpenSubtitles y del corpus CREA de la Real Academia Española (RAE).

Nótese que no hay pretensión de evaluar características adicionales de los paneles tales como la capacidad informativa de las imágenes, la tipografía o el contraste, así como no existe intención de comparar los museos entre sí.

\section{Análisis y resultados}

\subsection{Análisis y resultados de los indicadores clásicos}

El análisis de los textos indica que los carteles contienen 13,4 oraciones de media y 4,2 párrafos por cartel. Como se observa en la tabla 4, los paneles del Museo Nacional de Antropología presentan una extensión significativamente superior con una media de 29,3 oraciones y 6 párrafos por cartel.

El valor medio del número de palabras por cartel es de 188,2 . A este respecto resultan reveladores los datos del Museo Egipcio con un volumen inferior de palabras, y con datos próximos a los valores medios de oraciones y párrafos. Contrasta con el elevado número de palabras en los carteles de Museo del Modernismo con respecto al número de oraciones, lo que indica frases largas en estos textos.

\subsection{Resultados del test manual de comprensibilidad}

La evaluación manual de los participantes indica su percepción sobre el nivel de facilidad/dificultad del texto. Se han valorado en tres niveles: difícil, normal y fácil. A continuación, con el propósito de obtener sólo dos niveles (difícil y fácil) se preparó una batería de consultas que implican inferir información a través de los textos. El éxito en su respues-

Tabla 4. Valores medios de los textos de los paneles de los museos

\begin{tabular}{|l|c|c|c|}
\hline \multicolumn{1}{|c|}{ Museo } & Oraciones & Párrafos & Palabras \\
\hline Museo de la BNE & 9,7 & 3,9 & 230,1 \\
\hline Museo Nacional de Antropología & $\mathbf{2 9 , 3}$ & $\mathbf{6 , 3}$ & 208,9 \\
\hline Museo Nacional de Arqueología & 9,2 & 3,5 & 136,8 \\
\hline Museo Egipcio & 10,6 & 3,5 & 93,5 \\
\hline Museo de Historia & 7,5 & 3,7 & 180,7 \\
\hline Museo del Modernismo & 10 & 3,5 & $\mathbf{2 7 3 , 2}$ \\
\hline
\end{tabular}




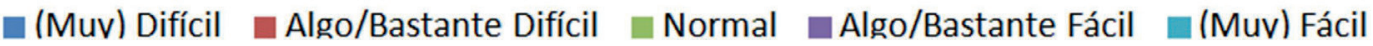

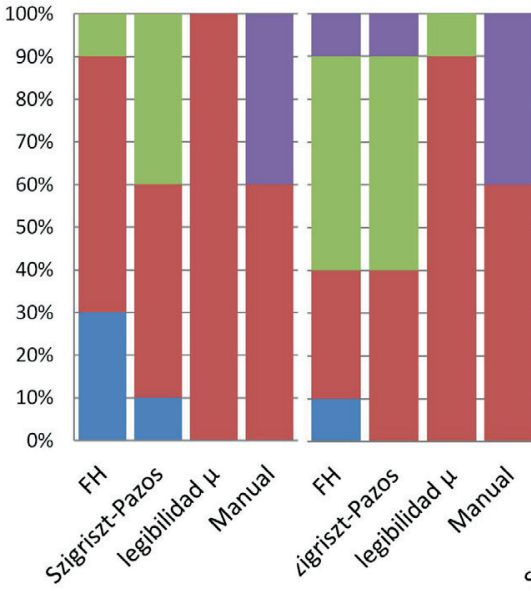

BNE
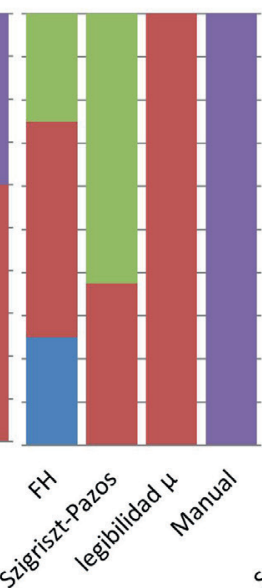

Museo Nac. Arqueología
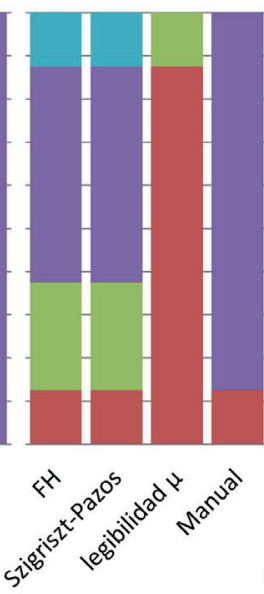

Museo Egipcio Barcelona
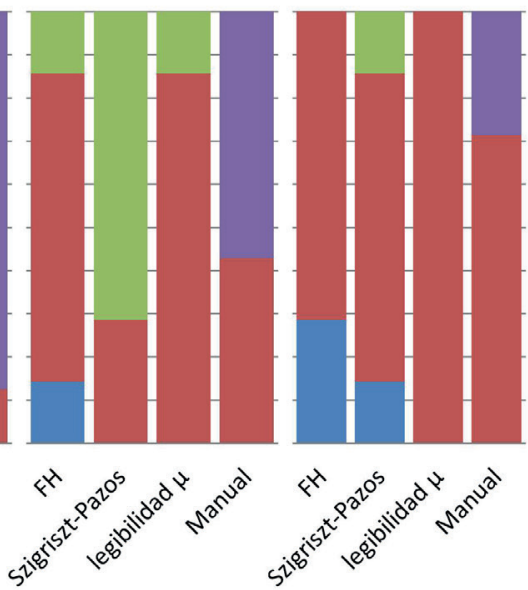

\section{Museo Historia} Madrid
Museo

Modernismo

Figura 1. Evaluación de las métricas clásicas para el español, calculados por Legible.es (todos los índices agrupados en 5 niveles, salvo el manual con dos niveles)

ta determina la dificultad del contenido. Estas consultas se han aplicado cuando la dificultad fue juzgada como normal o había una percepción contradictoria entre fácil y difícil. Los resultados se muestran en la figura 1 . Se ha obtenido un 0,421 de grado de acuerdo entre los múltiples evaluadores según la Kappa de Fleiss (Fleiss, 1971), lo que se corresponde con una concordancia media (Landis; Koch, 1977).

Los tiempos para leer y evaluar los textos en relación con el número de palabras del panel se muestran en la tabla 5 .

La correlación según Spearman entre el tiempo y las carac-

Tabla 5. Tiempos para leer y número de palabras del panel

\begin{tabular}{|l|c|c|}
\hline \multicolumn{1}{|c|}{ Museo } & $\begin{array}{c}\text { Tiempo para } \\
\text { leer (seg) }\end{array}$ & Palabras/seg \\
\hline Museo de la BNE & 80,3 & 3,15 \\
\hline Museo Nacional de Antropología & 75,0 & 3,11 \\
\hline Museo Nacional de Arqueología & 55,7 & 2,83 \\
\hline Museo Egipcio & 33,2 & 2,86 \\
\hline Museo de Historia & 60,3 & 2,25 \\
\hline Museo del Modernismo & 86,0 & 3,00 \\
\hline
\end{tabular}

terísticas del texto son significativas a un nivel 0,01 para el tamaño del párrafo $(0,52)$, número de oraciones $(0,57)$, nombres propios $(0,69)$, para infrecuentes según Freeling $(0,89)$ y para número de palabras $(0,96)$.

\subsection{Resultados de los tests clásicos de comprensibilidad}

La figura 1 también muestra los datos resultantes de la evaluación de comprensibilidad global de los textos de los 6 museos según las métricas clásicas para el español: FH, SP y Legibilidad $\mu$. Se ha obtenido una tasa de acuerdo significativa entre FH y Szigriszt-Pazos (con una medida de acuerdo de Kappa de 0,48). Por el contrario la métrica Legibilidad $\mu$ no muestra un acuerdo significativo con ninguna de las otras dos métricas (FH y SP).

\subsection{Resultados de los indicadores lingüísticos}

Mediante el procesamiento automático con Freeling se han identificado las categorías gramaticales de los términos de los paneles. Como se observa en la Tabla 6, destacan el porcentaje de adjetivos en el Museo de Historia y el Museo de Arqueología. Un número superior de nombres propios se localiza en los textos del Museo de Antropología, y el Museo del Modernismo. Y el valor más elevado en el uso de pronombres es

Tabla 6. Categorías morfológicas en los textos por museo (en porcentaje)

\begin{tabular}{|l|c|c|c|c|c|c|}
\hline \multicolumn{1}{|c|}{ Museo } & Adjetivos & Adverbios & Fechas & $\begin{array}{c}\text { Nombres } \\
\text { propios }\end{array}$ & Pronombres & Sustantivos \\
compuestas
\end{tabular}


Tabla 7. Valores medios para distintas categorías gramaticales para los documentos clasificados como fáciles y difíciles

\begin{tabular}{|l|c|c|}
\hline \multicolumn{1}{|c|}{ Atributo } & $\begin{array}{c}\text { Documentos } \\
\text { fáciles }\end{array}$ & $\begin{array}{c}\text { Documentos } \\
\text { difíciles }\end{array}$ \\
\hline Adjetivo calificativo (\%) & $\mathbf{9 , 2}$ & 7,1 \\
\hline Adjetivos ordinales (\%) & 0,1 & $\mathbf{0 , 2}$ \\
\hline Adverbios (\%) & 1,5 & 1,9 \\
\hline Nombres propios (\%) & 5,3 & $\mathbf{7 , 4}$ \\
\hline Pronombres de relativo (\%) & 1,4 & $\mathbf{1 , 8}$ \\
\hline Pronombres personales (\%) & 0,5 & 0,8 \\
\hline Pronombres indefinidos (\%) & 0,2 & $\mathbf{0 , 5}$ \\
\hline Sustantivo (\%) & $\mathbf{2 5 , 3}$ & $\mathbf{2 1 , 4}$ \\
\hline Palabras compuestas (\%) & 2,8 & $\mathbf{3 , 7}$ \\
\hline \multicolumn{2}{|c|}{} \\
\hline Promedio n palabras & 139,8 & $\mathbf{2 6 7 , 2}$ \\
\hline Promedio nº oraciones & 12,2 & $\mathbf{1 5 , 4}$ \\
\hline
\end{tabular}

para el Museo de la BNE, y en el Museo Egipcio en el caso de los sustantivos comunes. También se incluye el cómputo de palabras compuestas reconocidas por Freeling y se observan valores superiores para el Museo del Modernismo.

En la tabla 7 se muestra el porcentaje de los valores medios de cada atributo lingüístico respecto a los documentos fáciles o difíciles de la colección. También se muestran los valores medios del número de palabras y oraciones de los paneles.

La tabla 8 muestra los valores para el indicador de familiaridad (infrecuencia o rareza) del término. Se representa como porcentaje de palabras infrecuentes, de acuerdo con los valores usados por Freeling, Corpus de Referencia del Español Actual (CREA) de la Real Academia Española (RAE) y el listado de frecuencias basado en OpenSubtitles.

http://corpus.rae.es/creanet.html

https://www.opensubtitles.org/es

Según las métricas de Freeling, se ha establecido un umbral menor de -11 para considerarla infrecuente; en el caso de la colección de textos de películas de OpenSubtitles se ha optado por registrar los que obtienen un valor menor de 10. Si bien los corpus muestran diferencias, el Museo de la BNE
Tabla 8. Media de términos infrecuentes por museo en relación con el número de palabras, utilizando los listados de frecuencia de Freeling, CREA y OpenSubtitles

\begin{tabular}{|l|c|c|c|}
\hline \multicolumn{1}{|c|}{ Museo } & Freeling & CREA & OpenSubtitles \\
\hline Museo de la BNE & 46,2 & 25,5 & 7,1 \\
\hline Museo N. de Antropología & 50,1 & 33,5 & 10,3 \\
\hline Museo N. de Arqueología & 35,0 & 21,0 & 6,4 \\
\hline Museo Egipcio & 23,7 & 14,9 & 3,4 \\
\hline Museo de Historia & 45,0 & 25,8 & 7,7 \\
\hline Museo del Modernismo & 66,6 & 37,6 & 12,0 \\
\hline
\end{tabular}

muestra en general el vocabulario más familiar, y el de Antropología y Modernista el vocabulario más infrecuente o menos familiar. Nótese que de acuerdo con los datos mostrados en la Figura 1, los museos de Arqueología y Egipcio son los que poseen mayor proporción de textos fáciles, mostrando en la tabla 8 un menor número de términos infrecuentes.

\subsection{Elaboración del modelo}

Para desarrollar un modelo predictivo que permita estimar el nivel de comprensibilidad de un texto se analizaron los datos con el software de minería de datos Weka (Witten; Frank; Hall, 2011) y se experimentó con diversos algoritmos de clasificación (J48, PART, Random forest y Ramdom tree) con evaluación cruzada.

Se calculan los valores de referencia según las métricas e indicadores más referenciados. Tradicionalmente para estimar la mejora de los modelos de comprensión textual se emplea un nivel de referencia calculado con el número de palabras medio por oración (Larsson, 2006). Se crea un valor de referencia con los indicadores clásicos de comprensibilidad, basado en los datos obtenidos con la métrica de $\mathrm{FH}$ y el resultado de las evaluaciones manuales (valor de referencia FH). Los mejores datos surgen de la aplicación de los algoritmos $J 48$ y PART. Con J48, la predicción del modelo resulta del $38 \%$ de precisión y una medida- $F\left(F_{1}\right)$ ligeramente inferior al $50 \%(0,47)$. Se elabora otro valor de referencia basado en el indicador de tamaño de oración (valor de referencia: tamaño oración) con el que se obtiene una precisión del $54 \%$ y una $F_{1}$ del $55 \%$ (tabla 9 ).

A continuación, con los datos identificados de los procesa-

Tabla 9. Valores de los modelos con indicadores lingüísticos y de familiaridad terminológica. Como valores de referencia se ha tomado el cálculo de FH y el tamaño de oración.

\begin{tabular}{|c|c|c|c|}
\hline Modelo & Precisión & Exhaustividad & Medida- F \\
\hline $\begin{array}{l}\text { M1. Familiaridad término } \\
\text { Rareza según } C R E A \text {, Freeling y OpenSubtitles }\end{array}$ & 0,80 & 0,80 & 0,79 \\
\hline $\begin{array}{l}\text { M2. Lingüística } \\
n^{\circ} \text { palabras, nº palabras compuestas, adjetivos calificativos, adjetivos ordinales, adverbios, } \\
\text { sustantivos, nombres propios, pronombres personales, indefinidos y de relativo }\end{array}$ & 0,84 & 0,84 & 0,84 \\
\hline $\begin{array}{l}\text { M3. Combinado } \\
n^{\circ} \text { palabras, nº palabras compuestas, adjetivos calificativos, adjetivos ordinales, adverbios, } \\
\text { sustantivos, nombres propios, pronombres personales, indefinidos y de relativo } \\
\text { Rareza según CREA, Freeling y OpenSubtitles }\end{array}$ & 0,86 & 0,86 & 0,86 \\
\hline Valor de referencia: FH & 0,38 & 0,62 & 0,47 \\
\hline Valor de referencia: Tamaño oración & 0,54 & 0,56 & 0,55 \\
\hline
\end{tabular}


mientos de los textos de los paneles, se testeó un total de 40 indicadores, en múltiples iteraciones, de los que se seleccionaron 13 atributos. Se ha experimentado con diversos algoritmos automáticos de aprendizaje y aspectos lingüísticos para comprobar si es posible determinar el nivel de comprensibilidad (fácil o difícil) del texto de los paneles de museo. Se propone un modelo híbrido que incorpore las variables clásicas, de frecuencia y lingüísticas. Los indicadores que han sido seleccionados para estimar la dificultad/facilidad de los textos de los paneles de los museos han sido: 1) palabras compuestas, 2) pronombres de relativo, 3) infrecuentes Freeling, 4) número de palabras, 5) pronombres indefinidos, 6) sustantivos, 7) adverbios, 8) adjetivos calificativos, 9) adjetivos ordinales, 10) infrecuentes según OpenSubtitles, 11) infrecuentes según CREA, 12) nombres propios, y 13) pronombres personales. Todas ellas con t-Student significativamente diferentes para $p<0,05$ para los documentos sencillos y difíciles.

Existe una necesidad de incluir la familiaridad del término a las métricas de legibilidad

Estos atributos se analizaron y se combinaron para confeccionar tres modelos predictivos (tabla 8). En el primer caso, modelo 1 (M1), los indicadores de familiaridad del término. En el modelo 2 (M2) se incorporan los indicadores lingüísticos, y en el modelo 3 (M3) se combinan los indicadores clásicos, la familiaridad del término y los indicadores lingüísticos. Del mismo modo que para los valores de referencia, el algoritmo de clasificación aplicado ha sido el J48 con validación cruzada en diez carpetas utilizado en Weka. Junto a $J 48$ se probó el algoritmo PART, elegidos ambos por sus óptimos resultados y fácil interpretación, aunque $J 48$ obtuvo mejores métricas. Se han incorporado los indicadores basados en el uso de categorías gramaticales, para comprobar si puede repercutir y mejorar la estimación automática del nivel de comprensibilidad.

Los valores obtenidos con los diferentes modelos para la estimación de la comprensibilidad se muestran en la tabla 9. Para analizar si el modelo se comporta de un modo similar para determinar entre los dos conjuntos de comprensibilidad (fácil y difícil), se analiza en términos de precisión, exhaustividad y F1 el modelo que ha obtenido los mejores resultados, M3-Combinado (tabla 10 ).

\section{Discusión}

En relación con los resultados de estimación de la comprensibilidad según las métricas clásicas, se observa una escasa correspondencia entre algunas medidas. En la estimación automática destaca la valoración hacia la dificultad de los textos para ser comprendidos en las métricas $\mathrm{FH}, \mathrm{SP}$ y $L \mu$ para los textos del Museo de la BNE y del Museo del Modernismo. Los resultados obtenidos por $L \mu$ son destacables por cuanto se decanta en todos los casos hacia la dificultad de los textos para ser leídos, a pesar de estar en un lenguaje estándar y dirigido a todos los públicos. Además subraya la asignación casi exclusiva a la categoría difícil, en comparación con los valores observados manualmente.

Con respecto a los tiempos de lectura para su evaluación se observa que existe correspondencia entre el número de palabras, o nombres propios, o palabras familiares según Freeling, o nombres propios, y la estimación de dificultad. Y en consecuencia, cuanto mayor es el valor de estos indicadores, mayor percepción de dificultad para la comprensión del documento.

En cuanto a los resultados de las estimaciones manuales, se observa cómo los participantes declaran una mayor dificultad para entender los carteles del Museo de la BNE, el Museo Nacional de Antropología, y el Museo Modernista (en coincidencia con la estimación automática). Por el contrario, es destacable la unanimidad indicando la facilidad de comprensión de los textos del Museo Arqueológico y el Museo Egipcio.

Las diferencias entre los tiempos dedicados a la lectura puede estar también en la raíz de estas observaciones, pudiéndose concluir que textos narrativos largos y con más nombres propios implican una velocidad de lectura menor (museos de la BNE, Antropología y Modernista). Aquellos que tienen enumeraciones de objetos con metadatos (soporte, datación, tamaño, etc.) se procesan en un tiempo más reducido (p. e. Museo Egipcio).

\section{La baja comprensión lectora en España necesita que se preste atención a la difi- cultad de los textos}

En relación con los indicadores lingüísticos, se observa que el aumento en palabras compuestas, pronombres, número total de palabras, adverbios, número de oraciones, nombres propios o proporción de palabras no familiares está asociado a una mayor complejidad. Por otro lado el aumento de sustantivos y adjetivos calificativos se asocia a mayor simplicidad. Estos resultados están en la línea de los ya citados en Zeng-Treitler et al., 2007; Leroy; Endicott, 2011; 2012, entre otros.

\section{Conclusión}

El modelado de la comprensión de un texto es un tema complejo por el volumen de factores que afectan, la variabilidad en cuanto a niveles de lectura, tipos de lectores y técnicas de evaluación aplicadas. 
Las métricas clásicas de comprensibilidad se basan fundamentalmente en la longitud de la palabra y la oración, sin embargo son criterios que representan una solución limitada y poco adecuada para algunos idiomas. Estudios más recientes exploran nuevos indicadores lingüísticos que proporcionen una dimensión más amplia para estimar la comprensión textual.

Los textos de los paneles de los museos son consumidos por público de todas las edades, con una capacidad lectora diversa y un bagaje cultural diferente. Estos textos deben estar dirigidos a todos los públicos y por tanto todos los visitantes con competencia lectora deberían ser capaces de comprender la información que allí se expone sin una gran dificultad. Sin embargo, los resultados obtenidos, tanto con métricas automatizadas como con la evaluación manual, indican que existen textos que se perciben como difíciles, con la consecuente falta de comprensión.

Se propone un modelo híbrido que incorpore las variables clásicas, de frecuencia y lingüísticas y aspectos lingüísticos como consecuencia de los experimentos con diversos algoritmos automáticos de aprendizaje para comprobar si es posible determinar el nivel de comprensibilidad. En este trabajo hemos utilizado la lecturabilidad como una medida de la comprensibilidad, aunque obviamente existen otros factores, como soporte gráfico y contexto.

Los paneles informativos son uno de los tipos documentales que menos información ha recibido en el pasado

El análisis de los resultados muestra que hay espacio para la mejora. Elementos ya mencionados como la narratividad o la coherencia en el hilo discursivo podrían mejorar el modelo. No obstante, en gran medida las evaluaciones dependen del evaluador. Memoria, capacidad de inferencia o conocimientos previos implican que diferentes evaluadores asignarán diferentes valores. Eso supone un límite superior que no es posible franquear. Además la capacidad de comprensión depende de lo que el lector interprete, que se referencie información superflua o información que se da por sabida, lo cual dificulta la comprensión.

Como trabajo futuro nos proponemos ampliar el corpus y contrastarlo con otros corpus para verificar los indicadores que tienen mayor impacto en la comprensibilidad de los textos, incluyendo indicadores lingüísticos más avanzados. También consideramos relevante incidir en el análisis de la familiaridad de los términos y determinar qué casuística supone un mayor impacto en la percepción de la dificultad. Por último no podemos olvidar el impacto de las imágenes explicativas que acompañan al texto en el proceso de la comprensión de la información.

\section{Agradecimientos}

Agradecemos la dedicación de las personas que han participado en la evaluación de los textos. Este trabajo está financiado por el Ministerio de Economía, Industria y Competitividad de España, con el número CSO2017-86747-R y el Programa Salvador de Madariaga.

\section{Referencias}

Aluísio, Sandra; Gasperin, Caroline (2010). "Fostering digital inclusion and accessibility: The PorSimples project for simplification of portuguese texts". En: Procs of the NAACL HLT 2010 Young investigarors workshop on computational approaches to languages of the Americas, Los Angeles, California, June, pp. 46-53.

http://www.aclweb.org/anthology/W10-1607

Aluisio, Sandra; Specia, Lucia; Gasperin, Caroline; Scarton, Carolina (2010). "Readability assessment for text simplification". En: Fifth workshop on innovative use of NLP for building educational applications, pp. 1-9.

https://goo.gl/S8L2WJ

Badarudeen, Sameer; Sabharwal, Sanjeev (2010). “Assessing readability of patient education materials: current role in orthopaedics". Clinical orthopaedics and related research, v. 468 , n. 10 , pp. $2572-2580$.

https://doi.org/10.1007/s11999-010-1380-y

Blanco, Roi; Lioma, Christina (2012). “Graph-based term weighting for information retrieval". Information retrieval, v. 15, n. 1 , pp. $54-92$.

https://doi.org/10.1007/s10791-011-9172-x

Bott, Stefan; Rello, Luz; Drndarevic, Biljana; Saggion, Horacio (2012). "Can Spanish be simpler? LexSiS: Lexical simplification for Spanish". En: Proceedings of Coling 2012, pp. 357-374.

https://ac/web.org/anthology/C/C12/C12-1023.pdf

Carbon, Claus-Christian (2017). "Art perception in the museum: How we spend time and space in art exhibitions". i-Perception, v. 8, n. 1.

https://doi.org/10.1177/2041669517694184

Dale, Edgar; Chall, Jeanne S. (1948). "A formula for predicting readability". Educational research bulletin, v. 27, n. 1, pp. 11-28.

Elhadad, Noémie (2006). "Comprehending technical texts: Predicting and defining unfamiliar terms". En: AMIA Annual symposium proceedings, pp. 239-243.

https://www.ncbi.nlm.nih.gov/pubmed/17238339

España (2002). "Ley 34/2002, de 11 de julio, de servicios de la sociedad de la información y de comercio electrónico". $B O E$, n. 166, 12 julio.

https://www.boe.es/buscar/act.php?id=BOE-A-2002-13758

España (2013) “Ley 19/2013, de 9 de diciembre, de transparencia, acceso a la información pública y buen gobierno". $B O E$, n. 295, 10 diciembre.

https://www.boe.es/buscar/doc.php?id=BOE-A-2013-12887

Feng, Lijun; Jansche, Martin; Huenerfauth, Matt; Elhadad, Noémie (2010). "A comparison of features for automatic readability assessment". En: Procs of the $23^{\text {rd }}$ Int l conf on computational linguistics: Posters, pp. 276-284. http://dl.acm.org/citation.cfm?id=1944566.1944598

Ferrando-Belart, Vicky (2004). "La legibilidad: un factor fundamental para comprender un texto". Atención primaria, v. 34, n. 3, pp. 143-146.

https://goo.gl/E3S4Ko 
Fleiss, Joseph L. (1971). "Measuring nominal scale agreement among many raters". Psychological bulletin, v. 76, n. 5, pp. 378-382.

https://doi.org/10.1037/h0031619

François, Thomas; Fairon, Cédrick (2012). "An 'Al readability' formula for French as a foreign language". En: Joint conference on empirical methods in natural language processing and computational natural language learning, Jeju Island, Korea, 12-14 July, pp. 466-477.

http://www.ac/web.org/anthology/D12-1043

François, Thomas; Miltsakaki, Eleni (2012). “Do NLP and machine learning improve traditional readability formulas?". En: Proceedings of the First workshop on predicting and improving text readability for target reader populations, pp. 49-57.

http://dl.acm.org/citation.cfm ?id=2390916.2390925

Graesser, Arthur C.; McNamara, Danielle S.; Kulikowich, Jonna M. (2011). "Coh-Metrix: Providing multilevel analyses of text characteristics". Educational researcher, v. 40, n. 5, pp. 223-234.

https://doi.org/10.2307/2529310

Graesser, Arthur C.; McNamara, Danielle S.; Louwerse, Max M.; Cai, Zhiqiang (2004). "Coh-Metrix: Analysis of text on cohesion and language". Behavior research methods, instruments, \& computers, v. 36, n. 2, pp. 193-202.

https://doi.org/10.3758/BF03195564

Instituto de Turismo de España (2014). Museo del Prado. Caracterización de los visitantes. Encuesta a los visitantes del Museo del Prado.

https://goo.gl/k5c19g

Kauchak, David; Leroy, Gondy; Hogue, Alan (2017). “Measuring text difficulty using parse-tree frequency". Journal of the Asociation for Information Science and Technology, v. 68 , n. 9, pp. 2088-2100.

https://doi.org/10.1002/asi.23855

Keselman, Alla; Tse, Tony; Crowell, Jon; Browne, Allen; Ngo, Long; Zeng, Qing (2007). "Assessing consumer health vocabulary familiarity: An exploratory study". Journal of medical internet research, v. 9, n. 1, e5.

https://doi.org/10.2196/jmir.9.1.e5

Landis, J. Richard; Koch, G. Gary (1977). "The measurement of observer agreement for categorical data". Biometrics, v. 33, n. 1, pp. 159-174.

http://www.ncbi.nlm.nih.gov/pubmed/843571

Larsson, Patrik (2006). Classification into readability levels. Implementation and evaluation. Uppsala Universitet. Department of Linguistics and Philology.

https://goo.gl/YuTSvq

Leroy, Gondy; Endicott, James E. (2011). “Term familiarity to indicate perceived and actual difficulty of text in medical digital libraries". En: Xing, Chunxiao; Crestani, Fabio; Rauber, A. (eds.). Digital libraries for cultural heritage, knowledge dissemination, and future creation: $13^{\text {th }}$ Int l conf on Asia-Pacific digital libraries, Icadl 2011, Beijing, China, October 2427, v. 7008, pp. 307-310.

https://doi.org/10.1007/978-3-642-24826-9_38
Leroy, Gondy; Endicott, James E. (2012). “Combining NLP with evidence-based methods to find text metrics related to perceived and actual text difficulty". En: Proceedings of the $2^{\text {nd }} A C M$ Sighit Intl health informatics symposium, pp. 749-754.

https://doi.org/10.1145/2110363.2110452

Leroy, Gondy; Endicott, James E.; Kauchak, David; Mouradi, Obay; Just, Melissa (2013). "User evaluation of the effects of a text simplification algorithm using term familiarity on perception, understanding, learning, and information retention". Journal of medical internet research, v. 15, n. 7, e144.

Ministerio de Cultura (2011). Conociendo a nuestros visitantes. Estudio de público en museos del Ministerio de Cultura. Resumen ejecutivo. Ministerio de Educación, Cultura y Deporte.

https://goo.gl/Xhirta

Montanero-Fernández, Manuel (2004). “Cómo evaluar la comprensión lectora: alternativas y limitaciones". Revista de educación, v. 335, pp. 415-424.

https://dialnet.unirioja.es/servlet/articulo?codigo $=1066564$

Morato, Jorge; Llorens, Juan; Génova, Gonzalo; Moreiro-González, José-Antonio (2003). "Experiments in discourse analysis impact on information classification and retrieval algorithms". Information processing \& management, v. 39, n. 6 , pp. $825-851$.

https://doi.org/10.1016/S0306-4573(02)00081-X

Morato, Jorge; Sánchez-Cuadrado, Sonia; Moreno, Valentín; Moreiro-González, José-Antonio (2013). “Evolución de los factores de posicionamiento web y adaptación de las herramientas de optimización". Revista española de documentación científica, v. 36, n. 3, e018.

https://doi.org/10.3989/redc.2013.3.956

Newbold, Neil; McLaughlin, Harry; Gillam, Lee (2010). "Rank by readability: Document weighting for information retrieval". En: Cunningham, Hamish; Hanbury, Allan; Rüger, Stefan. Advances in multidisciplinary retrieval. IRFC. Lecture notes in computer science, v. 6107, pp. 20-30.

https://doi.org/10.1007/978-3-642-13084-7_3

Nomura, Misako; Nielsen, Gyda S.; Tronbacke, Bror (2010). Guidelines for easy-to-read materials. IFLA professional reports. https://www.ifla.org/files/assets/hq/publications/ professional-report/120.pdf

OECD (2016). Skills matter. Further results from the survey of adult skills. OECD Publishing. ISBN: 9789264258044 https://doi.org/10.1787/9789264258051-en

Padró, Lluís; Collado, Miquel; Reese, Samuel; Lloberes, Marina; Castellón, Irene (2010). "FreeLing 2.1: Five years of open-source language processing tools". En: Procs of the $7^{\text {th }}$ Intl conf on language resources and evaluation (LREC'10), pp. 931-936.

https://goo.gl/bpQb1P

Pitler, Emily; Nenkova, Ani (2008). "Revisiting readability: A unified framework for predicting text quality". In: Procs of the Conf on empirical methods in natural language processing, pp. 186-195.

http://dl.acm.org/citation.cfm?id=1613715.1613742 
Sigaud-Sellos, Pedro (2010). Aproximación a los conceptos de legibilidad y lecturabilidad: aplicación a la lectura de textos digitales. Pamplona: Universidad de Navarra.

https://goo.gl/2uh4K3

Social Science Consulting (2013). TextQuest. http://www.textquest.de/tq-man42.pdf

Stenner, A. Jackson (1996). "Measuring reading comprehension with the lexile framework". En: $4^{\text {th }}$ North American conf on adolescent/adult literacy. Washington, D.C. https://goo.gl/tVTSbC

Stenner, A. Jackson; Smith, Malbert; Burdick, Donald S. (1983). "Toward a theory of construct definition". Journal of educational measurement, v. 20, n. 4, pp. 305-316. https://doi.org/10.1111/j.1745-3984.1983.tb00209.x

Temnikova, Irina; Vieweg, Sarah; Castillo, Carlos (2015). "The case for readability of crisis communications in social media". In: Procs of the $24^{\text {th }}$ Intl conf on world wide web, pp. 1245-1250. https://doi.org/10.1145/2740908.2741718

Tonelli, Sara; Tran-Manh, Ke; Pianta, Emanuele (2012). "Making readability indices readable". En: Naacl-HLT 2012 Workshop on predicting and improving text readability for target reader populations, pp. 40-48.

http://www.aclweb.org/anthology/W12-2206
Van-Oosten, Philip; Tanghe, Dries; Hoste, Veronique (2010). "Towards an improved methodology for automated readability prediction". En: LREC 2010: Seventh conference on international language resources and evaluation, pp. 775-782.

http://hdl.handle.net/1854/LU-1055826

Venturi, Giulia; Bellandi, Tommaso; Dell'Orletta, Felice; Montemagni, Simonetta (2015). "NLP-based readability assessment of health-related texts: A case study on Italian informed consent forms". En: Procs of the $6^{\text {th }}$ intl workshop on health text mining and information analysis, pp. 131141.

http://www.aclweb.org/anthology/W15-2618

Witten, Ian H.; Frank, Eibe; Hall, Mark A. (2011). "Data mining: Practical machine learning tools and techniques" ( $3^{\text {rd }}$ ed.). San Francisco (USA): Morgan Kaufmann Publishers. ISBN: 9780123748560

Zeng-Treitler, Qing; Kim, Hyeoneui; Goryachev, Sergey; Keselman, Alla; Slaughter, Laura; Smith, Catherine-Arnott (2007). "Text characteristics of clinical reports and their implications for the readability of personal health records". Studies in health technology and informatics, v. 129, pp. 1117-1121.

http://ebooks.iospress.nl/publication/11151

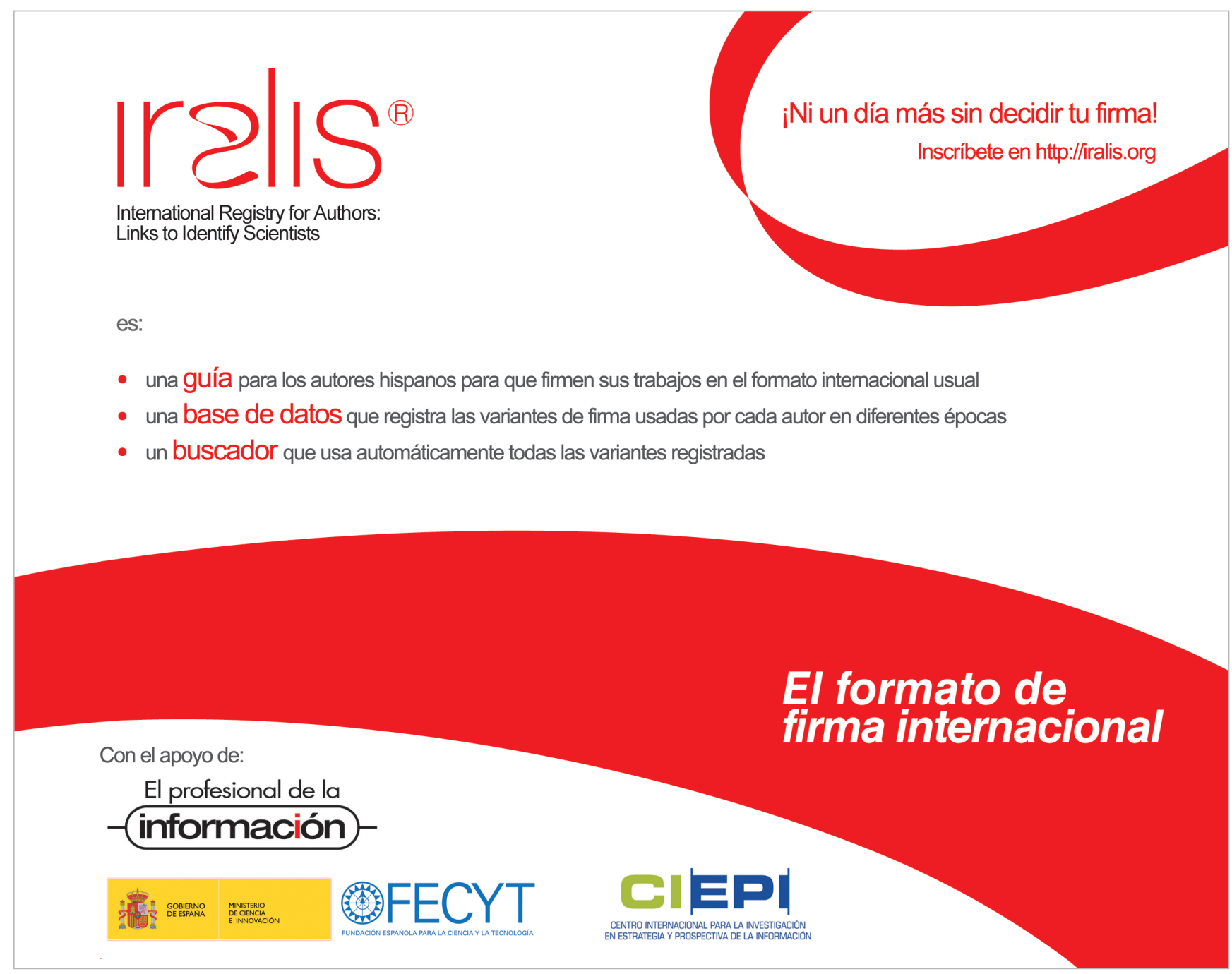

